Research Paper

\title{
Total mesopancreas excision for the treatment of pancreatic head cancer
}

\author{
Jingyong $\mathrm{Xu}^{1,2^{*}}$, Xiaodong Tian ${ }^{*}$, Yiran Chen ${ }^{1}$, Yongsu Ma1, Chang Liu1, Long Tian ${ }^{3}$, Jianwei Wang 3 , \\ Jianqiang Dong4, Di Cui ${ }^{5}$, Yang Wang6, Weiguang Zhang ${ }^{3 凶}$, Yinmo Yang1 ${ }^{1 凶}$ \\ 1. Department of General Surgery, Peking University First Hospital, 100034, Beijing, China; \\ 2. Department of General Surgery, Beijing Hospital, National Centre of Gerontology, China, 100730, Beijing, China; \\ 3. Department of Human Anatomy, Health Science Centre, Peking University, 100191, China; \\ 4. Department of Pathology, Peking University People's Hospital, Beijing, 100044, China; \\ 5. Department of Pathology, Beijing Hospital, National Center of Gerontology, China Beijing, 100730, China; \\ 6. Medical research and Biometrics Center, Fuwai Hospital, Peking Union Medical College, Beijing, 100730, China. \\ *Jingyong $\mathrm{Xu}$ and Xiaodong Tian contributed equally to this work. \\ $\triangle$ Corresponding authors:Yinmo Yang and Weiguang Zhang, Department of General Surgery, Peking University First Hospital, No. 8 Xishiku Street, \\ Dongcheng District, 100034, Beijing, China. E-mail:yangyinmo@263.net. Tel:+86-013601371285; Fax:+86-010-66119730 \\ (C) Ivyspring International Publisher. This is an open access article distributed under the terms of the Creative Commons Attribution (CC BY-NC) license \\ (https://creativecommons.org/licenses/by-nc/4.0/). See http://ivyspring.com/terms for full terms and conditions.
}

Received:2017.06.05; Accepted:2017.09.01; Published:2017.09.30

\begin{abstract}
Mesopancreas is a controversial structure. This study aimed to explore the anatomical characteristics of the mesopancreas, define the range of the total mesopancreas excision (TMpE), and evaluate the feasibility, safety and effectivity of TMPE in the treatment of pancreatic head cancer. The clinical and pathological data of 58 consecutive patients undergoing TMpE for pancreatic head carcinoma from January 2013 to December 2015 were analyzed prospectively. The perioperative morbidity, mortality and clinical outcomes of patients undergoing TMpE were compared with the patients undergoing conventional pancreaticoduodenectomy. The mesopancreas was located in the retropancreatic area, extending from the head, neck, and uncinated process of pancreas to the aorto-caval groove, in which there were loose areolar tissue, adipose tissue, nerve plexus, lymphatic and capillaries. We observed significantly higher R0 rate ( $94.8 \%$ vs. $81.4 \%, P=0.035)$, more lymph nodes ( 16.2 vs. $11.4, P=0.000)$, lower total and local recurrence rate (half-year local recurrence rate $7.8 \%$ vs. $23.7 \%, P=0.036$, one-year $18.2 \%$ vs. $39.5 \%, P=0.018)$ and longer disease-free survival ( 16.9 vs. 13.4 months, $P=0.044)$ in TMPE group than in control group. In conclusion, mesopancreas is different from mesorectum because there is no fascial envelop or anatomical boundary in this area. TMpE could be safely and feasibly performed for the treatment of pancreatic head cancer to increase the RO resection rate and improve the clinical outcomes.
\end{abstract}

Key words:Total mesopancreas excision; Pancreatic head cancer; Resection; Mesopancreas

\section{Introduction}

Pancreatic cancer is one of the most lethal human malignancies with very high mortality rate [1]. Surgery is the only potentially curative approach for pancreatic cancer if complete resection is possible, but the overall 5-year survival rate of all pancreatic cancer patients is still about $7 \%$ without significantly changes in the recent decades [2]. Nearly $80 \%$ of all adenocarcinomas of the pancreas occur in the head of the pancreas. Because of its special anatomical relationship with the mesenteric vascular and celiac artery, pancreatic head carcinoma has a high non-curative (R1 or R2) resection rate of approximately $24-51 \% \quad[3,4]$. Even with pancreaticoduodenectomy, the prognosis for the adenocarcinoma of the head of the pancreas remains poor, 5 -year survival rate is only $20 \%$ to $25 \%$ and median survival is between 10 and 20 months [2,5]. Local recurrence due to the incomplete removal at the 
site of resection, rather than to metastatic disease, is considered to be the primary reason for poor prognosis of patients with pancreatic head cancer [6-8]. Studies have shown that lymphogenic structures along the neuronal plexus posterior to the pancreas may play a key role in tumour cell infiltration, and the perineural tumour invasion rate is up to $77 \%$ in patients with pancreatic head cancer $[9,10]$. Thus it is important to improve the retropancreatic margin status to decrease loco-regional recurrence rate after surgery.

Total mesorectal excision (TME) has led to a significant decrease in the loco-regional recurrence rate for carcinoma of the rectum [10]. Mesopancreas was defined as a firm and well-vascularized structure extending from the posterior surface of the pancreatic head to behind the mesenteric vessels. Surgical resection of the structure as an intact entity comprising the pancreas and the mesopancreas might be correlated with a survival benefit for patients with pancreatic head cancer [10]. Meanwhile, mesopancreas was considered as the primary site for positive resection margins, which may account for the high rate of local recurrence in resectable pancreatic

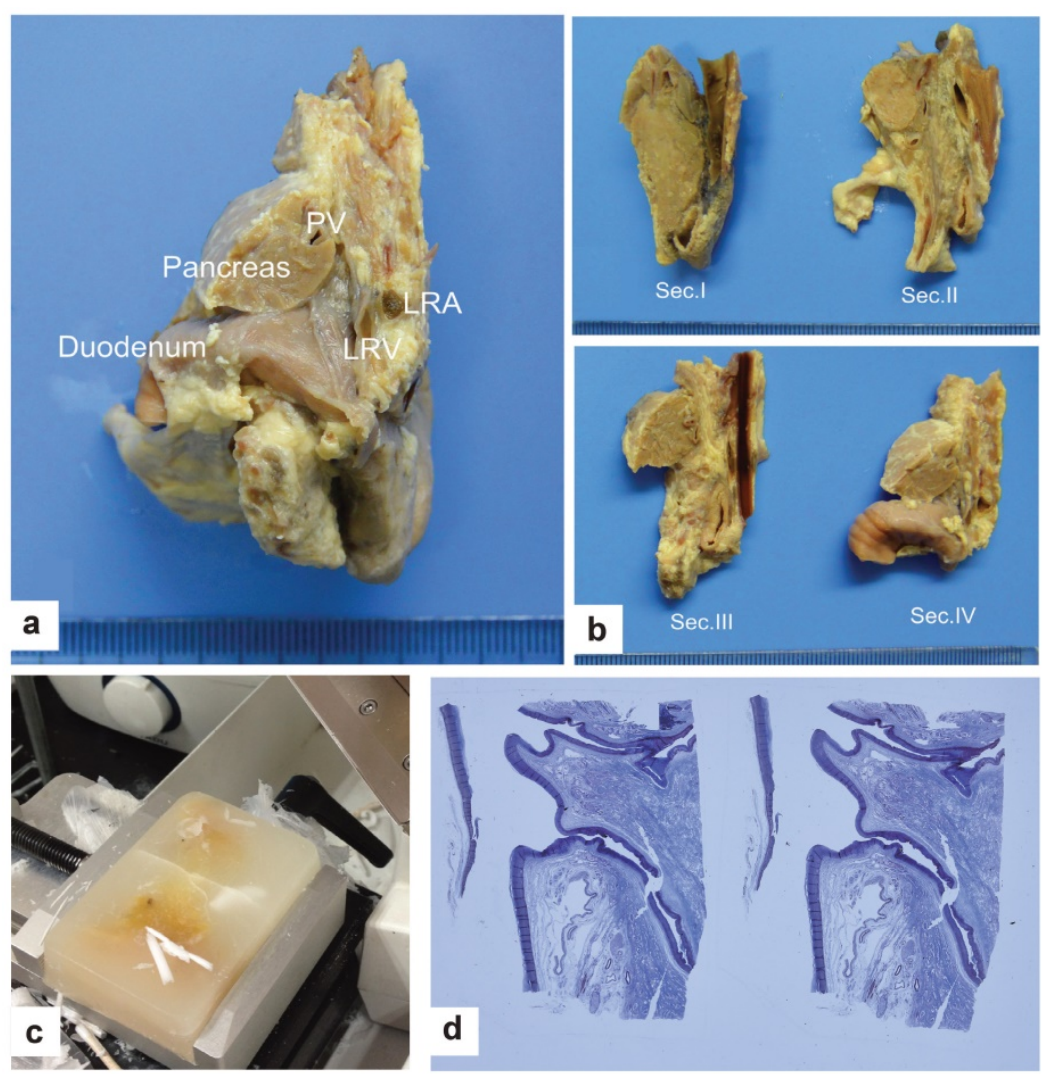

Figure 1. Preparation and examination of the specimens. a. Sagittal dissection along the line just left of the aorta in a child specimen (view from front to back). b. The cutting lines of the sagittal dissection in a child specimen (view from left to right). Section l:from the axis of the IVC along the middle portion; Section Il:from the middle of the tissues between the IVC and aorta; Section III:from the plane through the line of the CA and the SMA; Section IV:from the line of the CA and the SMA to the left edge of the aorta. c. Paraffin embedding and cutting $5 \mu$ m-thick slices. d. Representative H\&E staining. head cancers $[4,11]$. Accordingly, "total mesopancreas excision (TMpE)" was suggested to achieve a complete excision of the mesopancreas during pancreaticoduodenectomy, in order to increase the R0 resection rate for pancreatic head cancer [11,12]. However, the description of TMpE differed in previous studies [4,10-12]. Moreover, some anatomical studies questioned the existence of mesopancreas because of the absence of fibrous sheath or fascia in this area $[13,14]$.

Despite the controversies, the clinical appliance of $\mathrm{TMpE}$ on the improvement of $\mathrm{R} 0$ resection rate have been proven $[11,12,15]$. In this study, we aimed to define the anatomical boundary and contents of mesopancreas, and evaluate the feasibility, safety and effectiveness of TMpE for the treatment of pancreatic head cancer.

\section{Subjects and methods}

\section{Subjects}

This study was approved by the ethics committees of the Peking University First Hospital. Autopsy was made on six normal embalmed child cadavers, two fresh normal adult cadavers, four embalmed adult cadavers and two fresh cadavers of patients died from pancreatic head carcinoma. All cadavers were provided by the Department of Human Anatomy, Health Science Center of Peking University and the Centre of Body Donation of Peking University with legal formalities. To preserve the intact structures, especially the fascia layers, all organs containing the distal stomach, entire duodenum, proximal jejunum, common bile duct, pancreas, spleen and the related vessels were removed en bloc as deep as the tissues posterior to the aorta and inferior vena cava. The aorta and inferior vena cava were transected cranially at the upper edge of the coeliac artery and caudally at the lower edge of the renal vessels. Next, all specimens were fixed in $10 \%$ neutral formaldehyde, embedded in paraffin and subsequently cut into $5 \mu \mathrm{m}$ thin slices (Fig. 1). The sections were stained by haematoxylin and eosin (H\&E), Masson trichrome (Masson III) and Van Gieson (VG). S-100, CD-31 and D2-40 staining was performed to examine neurofibers, vessels and lymphangions, respectively. 


\section{Patients}

From January 2010 to December 2015, 101 consecutive pancreatoduodenectomies (PD) for resectable and borderline resectable cancer of the pancreatic head were performed at the Peking University First Hospital and Beijing Hospital. Between 2010 and 2012, conventional PD (CPD) was performed on 43 subjects as control group. Since January 2013, TMpE was introduced in our hospital and performed on 58 patients (TMpE group). The definition and difference between the procedures would be mentioned below. All patients were confirmed by pathologic examination. The patients who were given neoadjuvant therapy or who had no pancreatic duct adenocarcinoma or complete case records were excluded from the study. The same staff conducted the follow-ups. All patients provided informed consents.

\section{Surgical procedures}

For TMpE, an abdominal exploration was performed following an upper abdominal incision. An extended Kocher manoeuvre was done to the right and lower boundary of the SMA, and its origin on the aorta was dissected to evaluate the resectability. The gastrocolic ligament and greater omentum were then dissected to expose the anterior surface of the pancreas. The SMV was identified at the lower edge of the pancreas, and Henle's trunk and the inferior pancreatoduodenal vein were ligated. If SMV or PV was involved, the dissection of SMA would be the option which was described as "artery first". The hepatoduodenal ligament was skeletonized, the GDA was cut at its root on the CHA and tissue removal was performed along the CHA to its origin at the CA. After transecting the stomach or duodenum and jejunum, all the dissected tissues were pulled right and caudally. IPDA was recognized and ligated at its root either on the SMA or on the same trunk with the jejunal artery (JA), and the tissue around the IPDA was resected according to its root. Then, the tissue along the right hemi-circle of the SMA was dissected caudally to cranially and then along the right anterior portion of the aorta up to the origin of the CA. Meanwhile, standard lymphadenectomy (containing No.5, 6, 8a, 8p, 12b1, 12b2, 12c, 12p, 13a, 13b, 14a, 14b, $17 \mathrm{a}$ and $17 \mathrm{~b}$ groups according to the ISGPS consensus) was performed. Usually the specimen was removed en bloc except for Ln8p, 12p, which were dissected separately. In the cases with portal vein or SMV invasion, only if the tumour was resectable by pre-operative MDT evaluation, a negative margin could be achieved by gross judgement, an end-to-end vascular anastomosis could be done or the stenosis was less than $50 \%$ of the circle after partial resection of vascular wall, we would perform the vessel resection. However, artificial vascular replacement and artery resection were not recommended in our centre.

For borderline resectable cases, if negative margin could be achieved by gross judgement, we would perform extended TMpE including the resection of any extra-mesopancreatic structure, such as skeletisation of the anterior surface of the inferior vena cava (IVC), left renal vein (LRV) or aorta; whole circle dissection of the SMA or CA; and removal of tissues superior to the $\mathrm{CHA}$ to the diaphragm, and some extended lymphadenectomy (No.7, 9, 11, 14c, $14 \mathrm{~d}, 16 \mathrm{a} 2$ and16b1).

The main differences between CPD and TMpE were:(1) In CPD group, the operations were performed by different surgeons and no agreement or uniform standards reached with respect to the resection extent among the surgeons; in TMpE group, the operations were performed by the same surgeon group in each hospital, the procedure and resection extent reached consensus before the study and same standard would be followed in two hospitals and under cross quality check; (2) In TMpE group, the mesopancreatic root and the right semicircumference of SMA and CA plexus were dissected within the vascular sheath routinely, and furthermore, circumference plexus dissection of SMA would be performed for patients with borderline resectable tumours; In CPD group, however, mesopancreatic root was not revealed routinely, and the vascular sheath of SMA was usually not opened and the mesopancreatic tissues between pancreas and SMA, SMV were managed by bundle ligation which might result in suboptimal clearance and increased risk of $\mathrm{R} 1$ resection and recurrence.

\section{Clinical assessment}

The demographic characteristics, several serum biochemistry parameters at admission (PT, albumin, total and direct bilirubin), the rates of perioperative morbidity and mortality, pathological findings (histological type, positive resection margins and number of lymph nodes) and special procedures (vessel invasion and resection) were evaluated. A positive margin (R1) was defined as a tumour cell observed microscopically at the circumference margin of the transection independent of the mode of tumour spread. In the study group, the margins of specimen were sampled independently by the same group of pathologists and contained mesopancreatic root margin, posterior margin, portal vein margin, pancreatic transection margin, bile duct margin and others (like anterior surface and gastrointestinal margins).

To evaluate the feasibility and efficacy of the 
procedure, early and late clinical outcome parameters were recorded, including the amount of blood loss, the operation time, the total morbidity from the complications and the length of hospital stay.

The cases were followed up by a clinical visit or a phone call every 3 months. Subsequent adjuvant chemotherapy, the time of local recurrence, metastasis and mortality were recorded. Local recurrence rates at 6-month and 1-year were recorded. In order to exclude the influence of perioperative and non-tumour-related mortality, tumour-related disease-free survival (DFS) and overall survival (OS) were calculated.

\section{Statistical analysis}

The data were analyzed using IBM SPSS Statistics (Ver. 20.0, IBM Corp., Armonk, NY, USA). Continuous data with normal distribution were expressed as the means with standard deviation and analysed by an independent-sample $t$ test. Other continuous data were expressed as the median values with a range using the Wilcoxon rank-sum test. All categorized variables were compared using Fisher's exact test. The survival curves were calculated using

the Kaplan-Meier method, and survival rates were compared by the log-rank test. $\mathrm{P}$ value $<0.05$ was considered statistically significant.

\section{Results}

\section{Anatomical analysis of the mesopancreas}

In normal autopsy specimens, between the back of pancreas and aortocaval plane there were several parallel layers of collagen fibres called the Treitz's fusion fascia. From the lateral margin of the duodenum to the left margin of the aorta, the fascia extended continuously from the posterior surface of the pancreatic head and the third portion of the duodenum to behind the mesenteric vessels, which could be named "posterior lateral mesopancreas". Between the neck of the pancreas and the uncinate process of the pancreas to the superior mesenteric vessels and celiac artery, the tissues became denser than the fusion fascia which could be named "mesopancreatic root", and loose areolar tissue, adipose tissue, nerve plexus, lymphatics and capillaries were observed microscopically (Fig. 2). The tissues between the uncinate process and SMA extended left to the mesentery of jejunum without

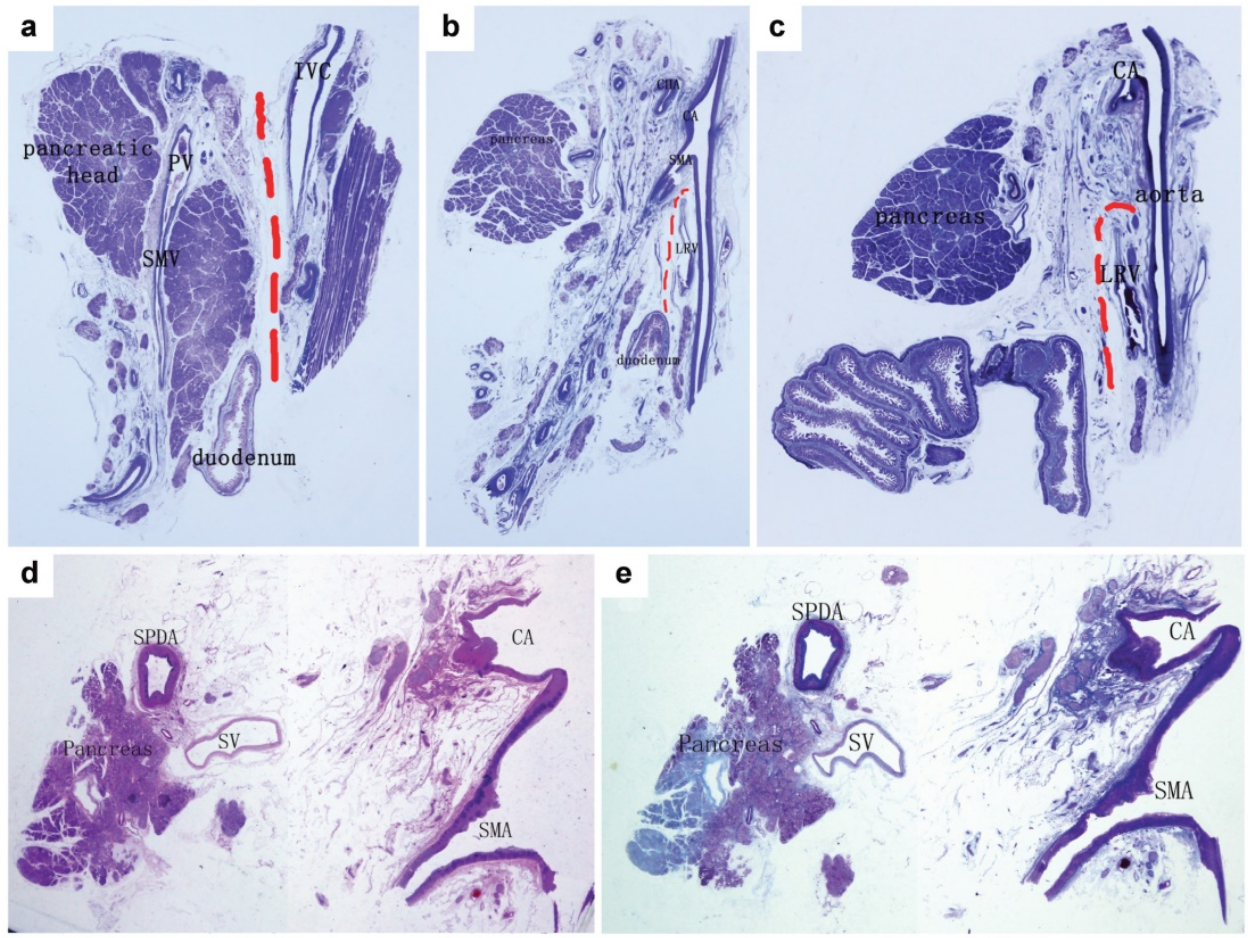

Figure 2. Analysis of a normal control specimen. a. A section along the IVC (Masson III staining). The red dotted line indicated that the fascia between the pancreatic head and inferior vena cava (IVC) was loose and could be easily dissected and that the fibrous layer near the pancreas was named "posterior lateral mesopancreas". b. A section along the aorta connecting the origins of the CA and the SMA (Masson III staining). c. A section beside the left margin of the aorta (Masson III staining). The fascia between the pancreas and the aorta was denser and contained vessels, nerves, lymphatics and several layers of fibres, which was defined as the "mesopancreatic root". The red lines showed a relatively looser space just beneath the SMA, and between the posterior lateral mesopancreas and the retroperitoneum. d. H\&E staining, and e. Masson III staining of a section from a normal adult specimen along the aorta connecting the origin of the CA and the SMA. The distance between the pancreas and the aorta was enlarged by fat tissue, and most of the structures of the mesopancreatic root distributed around the arteries. fibrous sheath or fascia was found to envelop the above structures.

In two cases of unresectable tumour specimens from who both suffered liver metastasis and common bile duct obstruction, CA and SMA were surrounded and infiltrated by the tumour both in the CT scan and pathological slices. Microscopically, intra-mesopancreatic nerves, lymph nodes, lymphangion and fascia fibres along the SMA and CA were found infiltrated by the tumour cells. However, via the sagittal observation, outside the origin of SMA, the tumour did not invade through the Treitz fusion fascia layers, which was complete 
and continuous anterior to the aortocaval plane. The metastasis of No. 16 lymph nodes was found, located outside the fascia layers (Fig. 3).

According to these results, CA and SMA could be considered the root and core of intra-mesopancreatic structures, surrounded by nerves, vessels and lymph tissues. CA and SMA could
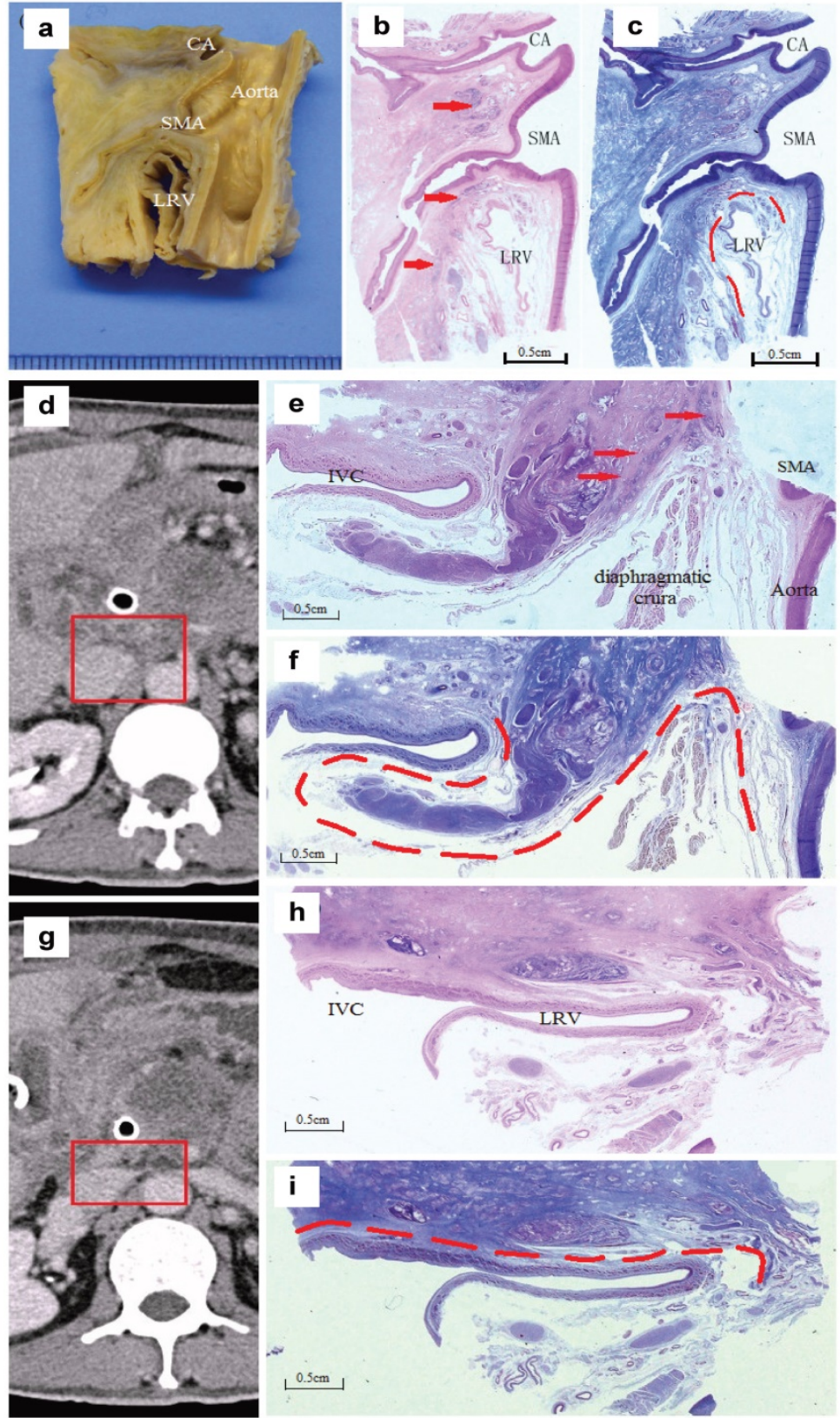

Figure 3. Analysis of specimen from unresectable pancreatic head cancer patients. a:Sagittal section of the tumour specimen dividing from the aorta connecting the origins of the $C A$ and the SMA. b:H\&E staining, the arrows indicated the front of the tumour invading along the SMA. c:Masson III staining, the dotted line showed the relatively intact fascia layers around the left renal vein (LRV). d. e. f:A section between the coeliac artery (CA) and the SMA (in the mesopancreas) $\mathrm{d}:$ The radiologic manifestation and the part adjusting to the specimen; e:H\&E, the arrows indicated the tumour cells invading along the nerve; f:Masson III, the dotted line showed the relatively intact fascia without tumour invasion. g. h. i:A section from the lower edge of the SMA to the middle of the left renal vein. g:The radiologic manifestation adjusting to the specimen; $h$ and i:(H\&E and Masson III). The dotted line showed an intact layer of fascia with compression but without tumour invasion. be involved in the tumour at earlier stage, which was the main reason of unresectability of the tumour. Otherwise, the posterior fusion fascia (posterior lateral mesopancreas) was rarely invaded, so Kocher's maneuverer could be finished during the operation in general. With this consideration, No.16 lymph nodes should be considered as an extra-mesopancreatic structure and regarded as distant metastasis if the tumour cells are involved.

Although there were no precise anatomical boundaries for mesopancreas, we proposed that mesopancreas could be regarded as an entity as bellow:the middle line along CA and SMA could be regard as left boundary, the second portion of duodenum as right boundary, the hepatic artery as superior boundary, lower edge of third duodenum as inferior boundaries, the anterior edge was the surface of pancreas, and the back edge contained two parts:the "posterior lateral mesopancreas", and the "mesopancreatic root" (Fig. 2). CA and SMA should be regarded as "intra-mesopancreatic structures" (Fig. 2). Meanwhile, lymph nodes around SMA (No. 14) were considered as the "intra-mesopancreatic structures", while lymph nodes between aorta and inferior vena cava (No. 16), which were outside the posterior lateral mesopancreas, should be considered as extra-mesopancreatic structure (Fig. 3).

Furthermore, we defined the total excision of mesopancreas within above extent as "standard $\mathrm{TMpE}$ " or "extent of Level I", and all the resected tissues could be considered within the mesopancreas (Fig. 4). We considered Level I TMpE as the standard and routine choice to treat pancreatic head carcinoma, and it has been implemented in our practice since 2013. Level II was defined as any procedure extending the extent of Level I and named as "extended TMpE", such as full-circle dissection of SMA or CA and clearance of No.16 lymph nodes. The decision regarding whether to perform Level II TMpE depended on the extent of tumour invasion. In order to get the negative margins, Level II TMpE would be frequently necessary, especially for some borderline resectable cases or some tumours from uncinate process. The Level II procedure was not a routine recommendation in our centre. 

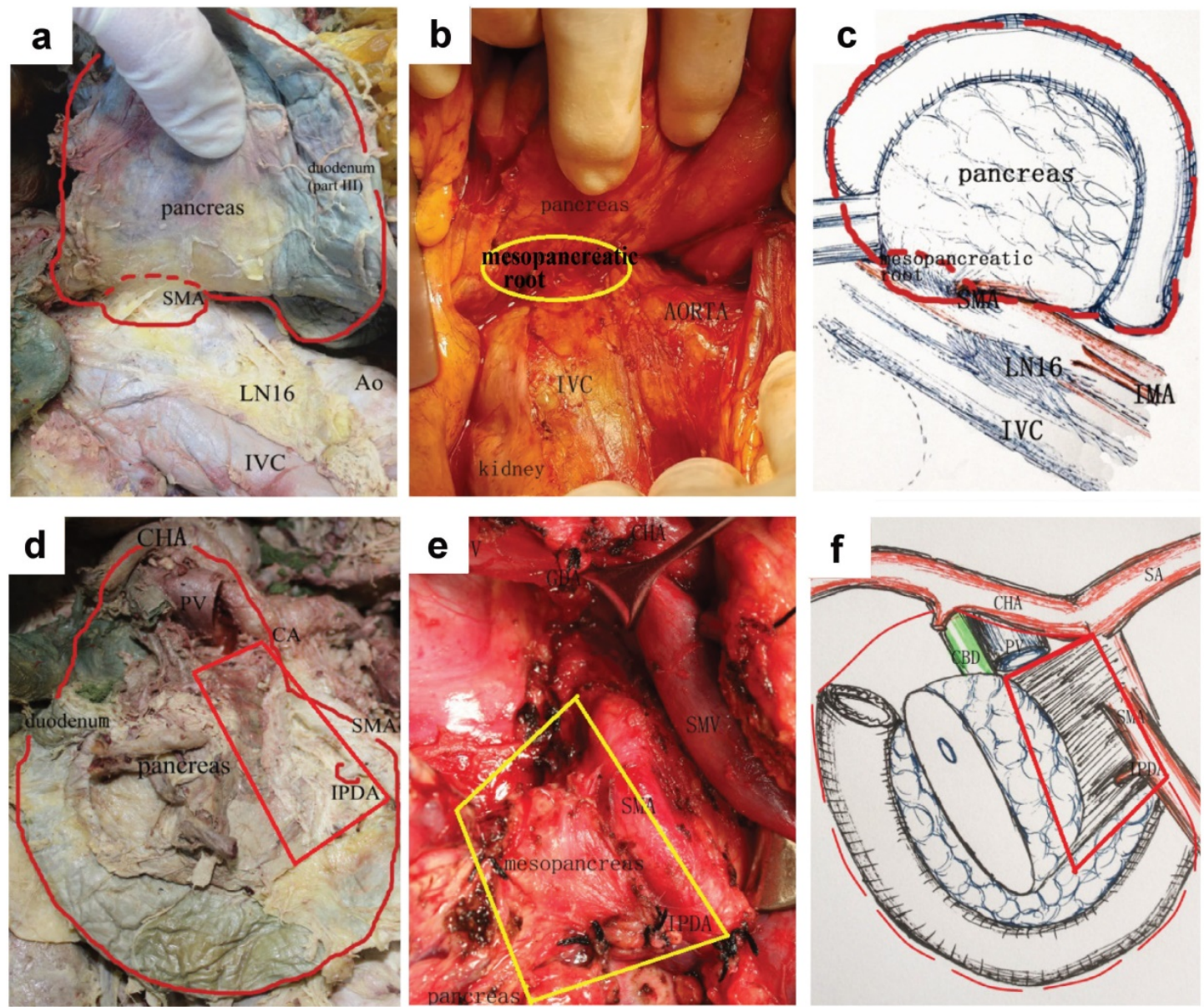

Figure 4. The anatomy and concept of a total mesopancreas excision (Level I). a. b \& c. Retropancreatic view via extended Kocher manoeuvre to dissect the posterior lateral mesopancreas. The red dotted line indicated the range of the TMpE. A small red or yellow circle indicated the mesopancreatic root. $d$, e \& $f$. Anterior view of the range of the TMPE. The mesopancreas presented as a quadrilateral structure.
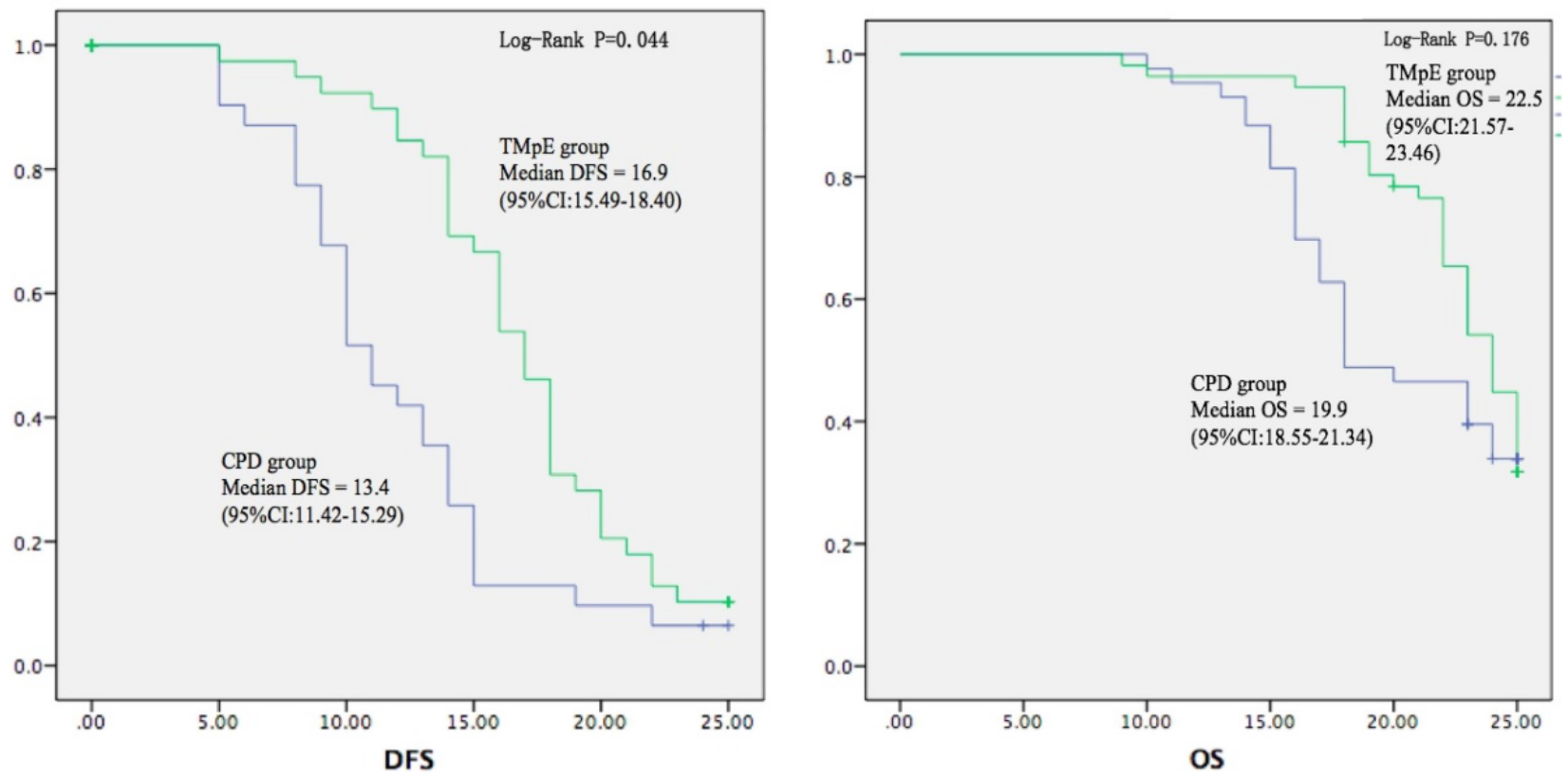

Figure 5. Postoperative tumour-related survival curves. The postoperative tumour-related disease-free and overall survivals (DFS and OS) were both longer in TMpE group than in CPD group, with a significant difference in DFS $(P=0.044)$. 


\section{Patient demographics and pathological characteristics}

There were no significant differences in age, gender, body mass index, morbidities from diabetes, ASA or several serum biochemistry parameters between TMpE and control groups (Table 1).

Table 1. Baseline data and pathologic parameters in two groups

\begin{tabular}{llll}
\hline & CPD (n=43) & TMpE (n=58) & P value \\
\hline Age (year) & $62.98 \pm 10.51$ & $63.31 \pm 9.91$ & 0.871 \\
BMI $\left(\mathrm{kg} / \mathrm{m}^{2}\right)$ & $23.07 \pm 3.58$ & $24.12 \pm 3.41$ & 0.219 \\
Gender $(\mathrm{M} / \mathrm{F})$ & $23 / 20$ & $40 / 18$ & 0.112 \\
Diabetes & $9(31.0 \%)$ & $18(45.0 \%)$ & 0.241 \\
ASA score (2/3) & $24 / 5$ & $35 / 5$ & 0.837 \\
TBIL(mmol/L) & $148.97 \pm 163.45$ & $149.09 \pm 124.20$ & 0.997 \\
DBIL(mmol/L) & $109.56 \pm 129.19$ & $95.74 \pm 83.39$ & 0.616 \\
Album $(\mathrm{g} / \mathrm{L})$ & $38.92 \pm 4.77$ & $41.18 \pm 4.55$ & 0.053 \\
Size of tumor (mm) & $34.35 \pm 15.01$ & $36.33 \pm 15.27$ & 0.518 \\
Histological type & & & 0.360 \\
High differentiated & $3(7.0 \%)$ & $6(10.3 \%)$ & \\
Middle differentiated & $28(65.1 \%)$ & $43(74.1 \%)$ & \\
Low differentiated & $11(25.6 \%)$ & $7(12.1 \%)$ & \\
Non differentiated & $1(2.3 \%)$ & $2(3.4 \%)$ & 0.041 \\
R0 resection rate & $76.7 \%(33 / 43)$ & $91.4 \%(53 / 58)$ & 0.000 \\
Number of LN resected & $11.39 \pm 2.48$ & $16.24 \pm 2.75$ & \\
\hline BMI=body mass index; TBIL=total bilirubin; DBIL $=$ direct bilirubin; LN=lymph \\
node.
\end{tabular}

The pathological characteristics of the two groups were shown in Table 1 . The tumour size, cases with positive lymph nodes and histological differentiation were comparable between the two groups. Between 2013 and 2015, the rate of R0 resection increased significantly $(91.4 \%$ vs. $76.7 \%$, $\mathrm{P}=0.041$ ), and the number of resected lymph nodes was significantly higher in TMpE group $(16.24 \pm 2.75$ vs. $11.39 \pm 2.48, \mathrm{P}=0.000)$. In the TMpE Group, the level
II procedure were performed in 13 cases, who underwent extended resection including the clearance of No. 16 lymph nodes and full-circle dissection of SMA or CA. Since the number of patients undergoing level II procedure was too small, we did not separate it from the TMpE group.

\section{Perioperative outcomes and follow-up}

Table 2 showed the intra-operative data of all 101 cases. The intra-operative blood loss and operation time were lower in the TMpE group without statistical significance. Considering that vessel resection might influence the amount of blood loss and surgery time, we analysed these two parameters in the subgroups of surgery with or without vessel resection. The results did not reveal any significance between two subgroups in these parameters. The length of the postoperative hospital stay was shorter in the TMpE group, and overall postoperative complication morbidities were reduced, although more extended resection procedure was performed compared to conventional group.

All the cases were followed up until December 31st, 2016 or death. 12 cases were lost. The rate of adjuvant chemotherapy was similar between the two groups. The local and total recurrence rates at 6-month and 1-year after surgery were lower in the TMpE group, and both the 6-month and 1-year local recurrence rates were significantly reduced in the TMpE group (Table 2). The postoperative tumour-related survivals (DFS and OS) were both longer in the TMpE group (Fig. 5), DFS was 16.9 months in the TMpE group (95\% CI:15.49-18.40) vs. 13.4 months in control group (95\% CI:11.42-15.29) $(\mathrm{P}=0.044)$.

Table 2. Perioperative and follow-up data in two groups

\begin{tabular}{lll}
\hline & CPD (n=43) & TMpE (n=58) \\
\hline Mean operation time (min) & $397.11 \pm 112.68$ & $368.60 \pm 92.48$ \\
Mean blood loss (ml) & $532.22 \pm 319.79$ & $461.38 \pm 184.49$ \\
Cases without vessel resection & $n=38$ & $n=48$ \\
Mean operation time (min) & $380.60 \pm 113.09$ & $357.39 \pm 83.11$ \\
Mean blood loss (ml) & $515.35 \pm 299.86$ & $436.67 \pm 188.73$ \\
Total complication & $22(51.2 \%)$ & $23(39.7 \%)$ \\
Fistula & $13(30.2 \%)$ & $15(25.9 \%)$ \\
Postoperative hospital stay (day) & $28.28 \pm 18.24$ & $25.66 \pm 21.76$ \\
Adjuvant therapy & $28(65.1 \%)$ & $43(74.1 \%)$ \\
Half year total recurrence rate & $13(34.2 \%)$ & $9(17.6 \%)$ \\
Half year local recurrence rate & $9(23.7 \%)$ & $4(7.8 \%)$ \\
one year total recurrence rate & $21(55.3 \%)$ & $15(31.8 \%)$ \\
one year local recurrence rate & $15(39.5 \%)$ & 8.080 \\
Median DFS (month) & $13.4(95 \% \mathrm{CI}: 11.42-15.29)$ & 0.116 \\
Median OS (month) & $19.9(95 \% \mathrm{CI}: 18.55-21.34)$ & 0.250 \\
\hline
\end{tabular}




\section{Discussion}

The pancreas originates from the endoderm of the foregut at the 5 th week of embryonic development and contains the ventral and dorsal anlagen. Like the other intraperitoneal organs, the pancreas is covered with two layers of peritoneum. The fusion of the ventral and dorsal pancreas occurs in the 6th week when the vessels primarily come from the coeliac artery. After the 6th week, the pancreas is carried along with the mesogastrium and lies in a transverse plane, with its head to the right embedded in the bend of the duodenum and its tail reaching the spleen on the left. At this time, the IPDA from the SMA grows along the groove between the duodenum and the pancreatic head and connects with the branches from the GDA. Therefore, coeliac artery and SMA are considered the intra-mesopancreatic and core structure like the superior rectal artery for the rectum, along which the nerves, fascia and lymphatic tissues are distributed. The mesentery of the pancreas then fuses with the adjacent peritoneum, and the pancreas is firmly fixed. Then, the pancreas becomes an "extra-peritoneal organ", and the mesopancreas become vague duo to the fusion.

Gockel et al. first proposed the term "mesopancreas" on the basis of successful clinical application of total mesorectal excision in rectal cancer [10]. The mesopancreas was considered to be the anatomical space bounded anteriorly by the posterior surface of the pancreatic neck, posteriorly by the pancreaticoduodenal coalescence fascia and medially by the mesenteric vessels. By their definition, the mesopancreas contains nerves, lymphatics and vessels. Later, only two relevant studies have been published, including total fifty-eight autopsy cases $[13,14]$. However, a fibrous sheath of fascia was not found around the structure in the area of the mesopancreas. Therefore, the presence of this sheath remains controversial.

In this study, first we examined the existence of the mesopancreas from an embryological view. The mesopancreas becomes vague and boundless after the organ's rotation during embryonic development. We found several parallel layers of fibrous fascia between the back of the pancreas and retroperitoneum, but no fibrous sheath around the structures. Based on autopsy studies, we speculated that the mesopancreas cannot be defined the same as the mesocolon or the mesorectum. Similar to inferior mesenteric artery for mesorectum, CA and SMA are intra-mesopancreatic and could be considered the main vessels entering the mesopancreas via the mesopancreatic root; but unlike the dissection at the origin of inferior mesenteric artery in total mesorectal excision, it is impossible to dissect CA and SMA in TMpE. Thus total mesopancreatic excision may be impossible for pancreatic head carcinoma. Several studies on clinical specimens supported our opinion. Peparini et al. highlighted the differences between the mesorectum and the mesopancreas based on anatomical findings from a series of 89 consecutive pancreaticoduodenectomies and 71 cases of total mesorectal excisions (TME) [16]. They concluded that compared with TME, no well-defined anatomic boundaries could be found around the pancreas, making it impossible to completely excise the mesopancreas en bloc.

Despite the controversy on the anatomical reality of the mesopancreas, the definition of the mesopancreas and the application of total mesopancreas excision (TMpE) have been accepted in some clinical practices. Because the retropancreatic area has been shown to be the most frequent site of R1 resection and the primary site of tumour local recurrence, $\mathrm{TMpE}$ could increase the $\mathrm{R} 0$ resection, but its impact on local or systemic recurrence and the survival benefit for patients have not been determined [15]. Due to the lack of a well-defined landmark about $\mathrm{TMpE}$, there is no universal consensus on the resection extent in clinical practice. To achieve $\mathrm{R} 0$ resection margin, the extended $\mathrm{TMpE}$ or extensive retropancreatic soft tissue dissection was performed "as far extended as possible" or "maximally" in some centres, including lymphadenectomy of the lymph nodes 16b1, 16a2 and around the SMA lymph nodes $14 \mathrm{~d}$. Additionally, some new definitions have been proposed, such as total meso-pancreatoduodenum excision (tMPDe), systemic mesopancreas dissection and others [12,17]. Kawabata et al. reported a higher R0 resection rate with tMPDe $(93 \%$ vs. $60 \%$ of standard PD group) [12]. However, randomized controlled trials or the meta-analyses could not demonstrate any survival benefit of the extend procedure, but instead indicated the development of complications such as refractory diarrhoea [18-21].

SMA and CA are intra-mesopancreatic structures that cannot be resected. Full-circle clearance of SMA may lead to several severe postoperative complications. In view of the results on embryological and anatomical studies, we believe that $\mathrm{TMpE}$ should be regarded as a surgical concept rather than an anatomical structure, and the definition of mesopancreas and the extent of TMpE should be combined with the clinical feasibility. We suggest a relatively clear range of $\mathrm{TMpE}$ and apply it in our practice in which Level I and Level II are defined. In our study, Level I TMpE or standard TMpE was mainly applied to resectable pancreatic head tumours and Level II or extended TMpE mainly to borderline 
resectable tumours. The extent of Level I TMpE plus standard lymphadenectomy is consistent with the standard resection which is advocated for pancreatic carcinoma by ISGPS [22]. Meanwhile, Level II TMpE is consistent with the extended resection in ISGPS standard [18].

Studies of the mesopancreas and TMpE have led to a better understanding of surgical anatomy which help increase the rate of $\mathrm{R} 0$ resection. In our study, the $\mathrm{R} 0$ resection rate of $\mathrm{TMpE}$ was $91.4 \%$ vs. $76.7 \%$ in the $\mathrm{CPD}$ group with significance $(\mathrm{P}=0.041)$. In addition, clear anatomical dissection brought the benefits of a shorter operation time, less blood loss and a lower morbidity from complications. Some modifications to the conventional surgical approach have been tried for the purpose of TMpE, including "hanging manoeuvre", "posterior approach", "anterior artery-first approach", "left posterior approach" and "mesopancreas first approach" [23-27]. It is reported that the modified surgical approach will help to determine the resectability through early detection of the relationship between the tumour and SMA and identify the aberrant right hepatic artery to prevent accidental injury, and the modified approaches help increase the $\mathrm{R} 0$ resection rate [28].

In our clinical study, a total of 101 cases were included, and 89 of the cases were followed up. We found a significantly lower 6-month and 1-year local recurrence rates $(7.8 \%$ vs. $23.7 \%, \mathrm{P}=0.036 ; 18.2 \%$ vs. $39.5 \%, \mathrm{P}=0.018$ ), and lower overall recurrence rates in $\mathrm{TMpE}$ group. FDS was significantly longer in TMpE group which might be due to lower local recurrence rate (16.9 months vs. 13.4 months, $\mathrm{P}=0.044)$, while the difference in OS was not significant. However, for the patients with pancreatic head cancer, an increase in survival by 3 months may be considered clinically significant.

Our study had several limitations. Firstly, the number of autopsy cases and the number of patients in TMpE group are small. Secondly, the study is retrospective and there is bias relating to the different period, improvement of both surgical skills and instruments. Thirdly, the follow-up of the TMpE group was relatively short. Fourthly, there were 13 cases with borderline resectable tumours in $\mathrm{TMpE}$ group, which were regarded as unresectable and would not undergo resection procedure. A randomized clinical trial is needed to further evaluate the clinical outcome of TMpE.

In conclusion, the mesopancreas is different from mesorectum since it has no fascial envelop and it should be regarded as a surgical concept rather than an anatomical structure. TMpE is safe and feasible for pancreatic head cancer and helps increase the R0 resection rate and improve the clinical outcomes.

\section{Acknowledgements}

We thank Mr. Zhi-Gang Zhang (Fuqiao Company, Tianjin, China) for his valuable suggestion of creating large slices and Dr. Bin Zhang (Institution of Medical Information, Chinese Academy of Medical Science) for helping with the bibliography. This study was funded by "foundation of capital characteristic clinic project" from the Beijing Science and Technology Commission, China (No. Z131107002213043).

\section{Competing Interests}

The authors have declared that no competing interest exists.

\section{References}

1. Siegel RL1, Miller KD, Jemal A. Cancer Statistics, 2017. CA Cancer J Clin. 2017 Jan;67(1):7-30. doi:10.3322/caac.21387.

2. Salem AI, Alfi M, Winslow E, et al. Has survival following pancreaticoduodenectomy for pancreas adenocarcinoma improved over time? J Surg Oncol. 2015 Nov;112(6):643-9.

3. Fatima J, Schnelldorfer T, Barton J, et al. Pancreatoduodenectomy for ductal adenocarcinoma:implications of positive margin on survival. Arch Surg. 2010, 145(2):167-72. doi:10.1001/archsurg.2009.282.

4. Gaedcke J, Gunawan B, Grade M, et al. The mesopancreas is the primary site for R1 resection in pancreatic head cancer:relevance for clinical trials. Langenbecks Arch Surg 2010; 395:451-458.

5. Griffin JF, Poruk KE, Wolfgang CL. Pancreatic cancer surgery:past, present, and future. Chin J Cancer Res. 2015, 27(4):332-48. doi:10.3978/j.issn.1000-9604.2015.06.07.

6. Esposito I, Kleeff J, Bergmann F, et al. Most pancreatic cancer resections are R1 resections. Ann Surg Oncol 2008; 15:1651-1660.

7. Verbeke CS, Leitch D, Menon KV, et al. Redefining the R1 resection in pancreatic cancer. Br J Surg 2006; 93:1232-1237. doi:10.1002/bjs.5397.

8. Hishinuma S, Ogata Y, Tomikawa M, Ozawa I, Hirabayashi K, Igarashi S. Patterns of recurrence after curative resection of pancreatic cancer, based on autopsy findings. I Gastrointest Surg 2006; 10:511-518. doi:10.1016/j.gassur.2005.09.016.

9. Neoptolemos JP, Stocken DD, Dunn JA, et al. Influence of resection margins on survival for patients with pancreatic cancer treated by adjuvant chemoradiation and/or chemotherapy in the ESPAC-1 randomized controlled trial. Ann Surg. 2001, 234(6):758-68.

10. Gockel I, Domeyer M, Wolloscheck T, Konerding MA, Junginger T. Resection of the mesopancreas (RMP):a new surgical classification of a known anatomical space. World J Surg Oncol 2007; 5:44. doi:10.1186/1477-7819-5-44.

11. Adham M, Singhirunnusorn J. Surgical technique and results of total mesopancreas excision (TMpE) in pancreatic tumors. Eur I Surg Oncol 2012; 38:340-345. doi:10.1016/j.ejso.2011.12.015.

12. Kawabata $\mathrm{Y}$, Tanaka $\mathrm{T}$, Nishi $\mathrm{T}$, et al. Appraisal of a total meso-pancreatoduodenumexcision with pancreaticoduodenectomy for pancreatic head carcinoma. Eur J Surg Oncol 2012;38:574-9.

13. Agrawal MK, Thakur DS, Somashekar U, et al. Mesopancreas:myth or reality? JOP 2010; 5:230-233.

14. Bouassida M, Mighri MM, Chtourou MF, et al. Retroportal lamina or mesopancreas? Lessons learned by anatomical and histological study of thirty-three cadaveric dissections. Int J Surg 2013; 11:834-836.

15. Wu W, Wang $X, W u X$, et al. Total mesopancreas excision for pancreatic head cancer:analysis of 120 cases. Chin J Cancer Res. 2016, 28(4):423-8. doi:10.21147/j.issn.1000-9604.2016.04.05.

16. Peparini N, Caronna R, Chirletti P. The "meso" of the rectum and the "meso" of the pancreas:similar terms but distinct concepts in surgical oncology. Hepatobiliary Pancreat Dis Int 2015; 14:548-551. doi:10.1016/S1499-3872(15)60417-9.

17. Inoue $Y$, Saiura A, Yoshioka $R$, et al. Pancreatoduodenectomy with systematic mesopancreas dissection using a supracolic anterior artery-first approach. Ann Surg 2015; 262:1092-1101. doi:10.1097/SLA.0000000000001065.

18. Tol JA, Gouma DJ, Bassi C, et al. Definition of a standard lymphadenectomy in surgery for pancreatic ductal adenocarcinoma:a consensus statement by the International Study Group on Pancreatic Surgery (ISGPS). Surgery 2014; 156:591-600. doi:10.1016/j.surg.2014.06.016.

19. Michalski CW, Kleeff J, Wente MN, et al. Systematic review and meta-analysis of standard and extended lymphadenectomy in pancreaticoduodenectomy for pancreatic cancer. Br J Surg 2007; 94:265-273. doi:10.1002/bjs.5716.

20. Iqbal $\mathrm{N}$, Lovegrove RE, Tilney HS, et al. A comparison of pancreaticoduodenectomy with extended pancreaticoduodenectomy:a 
meta-analysis of 1909 patients. Eur J Surg Oncol 2009; 35:79-86. doi:10.1016/j.ejso.2008.01.002.

21. Jang JY, Kang MJ, Heo JS, et al. A prospective randomized controlled study comparing outcomes of standard resection and extended resection, including dissection of the nerve plexus and various lymph nodes, in patients with pancreatic head cancer. Ann Surg 2014; 259:656-664. doi:10.1097/SLA.0000000000000384.

22. Hartwig W, Vollmer CM, Fingerhut A, et al. Extended pancreatectomy in pancreatic ductal adenocarcinoma:definition and consensus of the International Study Group for Pancreatic Surgery (ISGPS). Surgery. 2014, 156(1):1-14. doi:10.1016/j.surg.2014.02.009.

23. Pessaux P, Rosso E, Panaro F, et al. Preliminary experience with the hanging maneuver for pancreaticoduodenectomy. Eur J Surg Oncol 2009; 35:1006-1010. doi:10.1016/j.ejso.2009.04.009.

24. Moldovan SC, Moldovan AM, Dumitraæcu T, et al. The advantages of retropancreatic vascular dissection for pancreatic head cancer with portal/superior mesenteric vein invasion:posterior approach pancreatico-duodenectomy technique and the mesopancreas theory Chirurgia (Bucur). 2012; 107:571-578.

25. Inoue Y, Saiura A, Yoshioka R, et al. Pancreatoduodenectomy with systematic mesopancreas dissection using a supracolic anterior artery-first approach. Ann Surg 2015; 262:1092-1101. doi:10.1097/SLA.0000000000001065.

26. Aimoto T, Mizutani S, Kawano $Y$, et al. Left posterior approach pancreaticoduodenectomy with total mesopancreas excision and circumferential lymphadenectomy around the superior mesenteric artery for pancreatic head carcinoma. J Nippon Med Sch 2013; 80:438-445. doi:10.1272/jnms.80.438.

27. Lupaşcu C, Andronic D, Grigorean VT, et al. Mesopancreas first dissection during pancreaticoduodenal resection:selective approach or paradigm? Hepato-Gastroenterology 2014; 61:463-468.

28. Chowdappa R, Challa VR. Mesopancreas in pancreatic cancer:where do we stand - review of literature. Indian J Surg Oncol 2015; 6:69-74. doi:10.1007/s13193-014-0294-7. 